\title{
Guest Editorial: Mathematics and Image Analysis
}

\author{
Jalal Fadili $^{1}$ • Gitta Kutyniok ${ }^{2}$ - Gabriel Peyré ${ }^{3}$ - Gerlind Plonka-Hoch ${ }^{4}$. \\ Gabriele Steidl $^{5}$
}

Published online: 18 March 2015

(C) Springer Science+Business Media New York 2015

This special issue highlights some recent developments in the field of mathematical image analysis. The emphasis of the issue is on the interplay between advanced mathematical methods (such as non-smooth optimization, variational methods and regularization, inverse problems, and partial differential equations) and their application to various problems in image processing and computer vision, including restoration, segmentation, and classification. The special issue comprises nine papers which cover a wide range of topics in mathematical image analysis as outlined below.

Jalal Fadili

Jalal.Fadili@greyc.ensicaen.fr

Gitta Kutyniok

kutyniok@math.tu-berlin.de

Gabriel Peyré

peyre@ceremade.dauphine.fr

Gerlind Plonka-Hoch

plonka@math.uni-goettingen.de

Gabriele Steidl

steidl@mathematik.uni-kl.de

1 GREYC, CNRS-ENSICAEN-University of Caen, 6 Bd Maréchal Juin, 14050 Caen Cedex, France

2 Technical University of Berlin, Str. des 17. Juni 136, 10623 Berlin, Germany

3 CNRS and CEREMADE, University of Paris-Dauphine, Place du Maréchal de Lattre de Tassigny, 75775 Paris Cedex 16, France

4 University of Göttingen, NAM, Lotzestr. 16-18, 37083 Göttingen, Germany

5 Department of Mathematics, University of Kaiserslautern, Paul-Ehrlich Str. 31, 67653 Kaiserslautern, Germany
- Variational imaging:

The paper "Preconditioned Douglas-Rachford algorithms for TV and TGV regularized variational imaging problems" (DOI JMIV-D-14-00124), by Bredies and Sun, applies the preconditioned Douglas-Rachford scheme, introduced previously by the same authors, to solve structured saddle-point problems involving total variation (TV) and total generalized variation (TGV) regularizers. A sublinear $O(1 / k)$ ergodic convergence rate is also established for the restricted primal-dual gap. The authors propose effective preconditioners for variational imaging problems such as TV denoising, TV deblurring, and TGV denoising.

In "Retinex by Higher Order Total Variation $L_{1}$ Decomposition" (DOI JMIV-D-14-00125), Liang and Zhang deal with the reflectance-illumination decomposition of images. Based on a Retinex approach and the observation that the illumination varies more smoothly than the reflectance, they propose a convex optimization model with an infimal convolution regularizer which incorporates first- and second-order derivatives.

- Inverse problems:

Faridani and Hass, in "On Numerical Analysis of View Dependent Derivatives in Computed Tomography" (DOI JMIV-D-14-00128), explore several existing numerical approximations of a view-dependent derivative which occurs in $\pi$-line inversion formula of $2 \mathrm{D}$ and $3 \mathrm{D} \mathrm{X}$ ray tomography. The discretization of this derivative is known to be the source of decrease of reconstruction quality and motivates this work. The authors have given a systematic analysis of this problem by providing a general common framework in which several discretization schemes of the literature are examined. The main results consist of determining the error terms for each scheme. 
The paper "Texture Generation for Photoacoustic Elastography" (DOI JMIV-D-14-00126) by Glatz, Scherzer, and Widlak proposes a technique for combining elastographic imaging with photoacoustics. The basic idea is to introduce textures into the solution by convolving the original inverse problem solution with a radial basis function. The paper shows how this can be done without/before actually solving the inverse problem.

Briskman and Needell, in "Block Kaczmarz Method with Inequalities" (DOI JMIV-D-14-00121R2), introduce and analyze a block version of the randomized Kaczmarz algorithm for solving mixed linear systems that involve both equality and inequality constraints. This class of randomized algorithms has potential to be of interest to practitioners working on image processing.

In "Numerical methods for parameter estimation in Poisson data inversion" (DOI JMIV-D-14-00122), Zanni et al. deal with hyperparameter estimation when solving regularized linear inverse problems with Poisson noise. More precisely, when a penalized variational approach is considered for Poisson data restoration, a regularization parameter has to be estimated, typically through a discrepancy principle. An alternative consists in considering the constrained form instead of the penalized one. In this work, the two forms are compared from the computational point of view, and numerical solutions to both of them are discussed.

- Segmentation and classification:

The paper "Global binary optimization on graphs for classification of high dimensional data" (DOI JMIV-
D-14-00123), by Merkurjev et al., describes a pair of splitting algorithms for solving a convex relaxation of a binary total variation + fidelity constraint program appropriate for binary semi-supervised classification of graph vertices. For this case, it is shown that the convex relaxation is indeed tight, hence providing guaranteed global minimizers of the original problem. The authors also describe extensive numerical experiments and comparison showing the benefits of convex relaxation.

In "Globally Optimal Joint Image Segmentation and Shape Matching based on Wasserstein Modes" (DOI JMIV-D-14-00132), Schmitzer and Schnörr adapt methods from optimal transport to model shapes seen as uniform distributions. This defines a Riemannian geometry on the space of 2-D shapes, which can in turn be used to solve various imaging problems, such as for instance segmentation.

In the paper "Explicit embeddings for nearest neighbour search with Mercer kernels" (DOI JMIV-D-14-00127), by Bourrier et al. proposes an explicit embedding scheme for nearest neighbor search using Mercer kernels. The method is split into two steps. Firstly, an explicit embedding is computed to map the initial space into a Euclidean space. Secondly, standard encoding on the Euclidean distances is applied. The proposed method, albeit very simple, has several advantages when compared with previous schemes especially for those relying on implicit embeddings. 